\title{
TG06
}

\section{Applied Rock Mechanics in Developing Tight Gas} Reservoirs

\author{
H. Abass* (Saudi Aramco)
}

\section{SUMMARY}

The experimental results indicate that the tensile fractures are much less conductive than shear fractures and the shear fractures are less conductive than propped fractures. The concept of effective stress within the rock matrix is totally different than that of natural fractures; therefore, the effective stress function for both matrix and natural fractures should be separately evaluated to obtain representative functions for any simulation study. The tensile fractures lose conductivity at very early stages of reservoir depletion.

Recommendations to manage these tensile fractures for optimum hydrocarbon recovery are suggested. 
Most tight gas reservoirs are stress-sensitive in which the rock matrix and both natural and created hydraulic fractures contribute to the hydrocarbon transport in a very complex manner. Understanding how the matrix frame, natural fractures, and created hydraulic fractures, behave as a function of stress, is vital to designing optimum stimulation treatments which are essential to produce these reservoirs economically.

Tight rock samples were tested to determine the permeability of matrix and fractures (natural and induced) as a function of effective stress. A naturally shear-fractured core was included, while a tensile natural fracture was simulated by splitting one core. The split core was then propped with small-mesh proppant to simulate a hydraulic fracture. Simulation runs were made to show the Productivity Index of various porous components as a function of time for two cases where the stressdependant permeability is considered and ignored. The optimum number of hydraulic fractures required to deplete a given drainage area is discussed.

The experimental results indicate that the tensile fractures are much less conductive than shear fractures and the shear fractures are less conductive than propped fractures. The concept of effective stress within the rock matrix is totally different than that of natural fractures; therefore, the effective stress function for both matrix and natural fractures should be separately evaluated to obtain representative functions for any simulation study. The tensile fractures lose conductivity at very early stages of reservoir depletion. Recommendations to manage these tensile fractures for optimum hydrocarbon recovery are suggested.

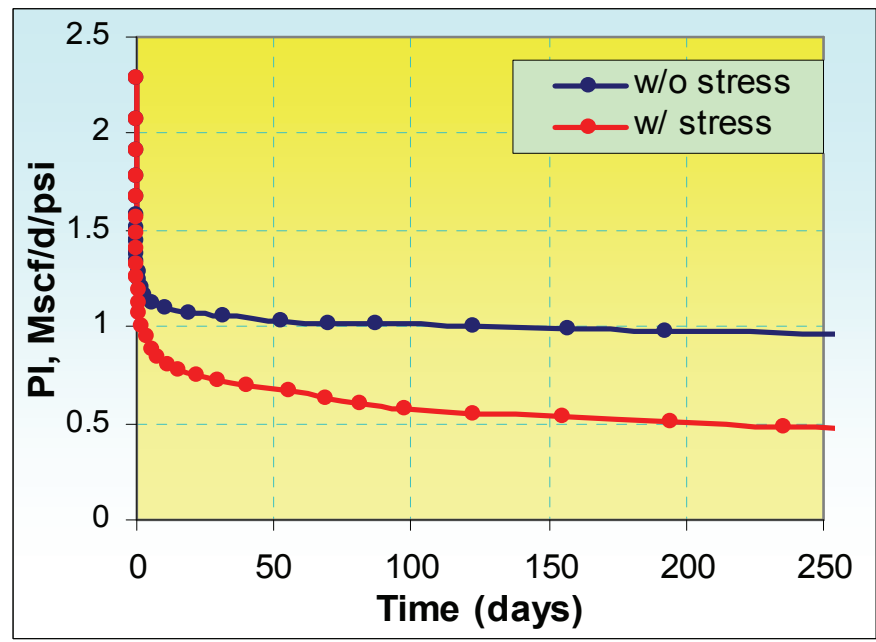

The stress effect is responsible for 50\% loss of the PI, which can be even more at higher production rate. 